\title{
Severity of Glaucoma at First Presentation to Hospital Eye Services
}

\author{
AYM El-Medany ${ }^{1}$, V. Swetha E. Jeganathan ${ }^{2 *}$ and Andrew J. Tatham ${ }^{3,4}$ \\ ${ }^{1}$ University Hospital Ayr Scotland, UK \\ ${ }^{2}$ W.K. Kellogg Eye Center, University of Michigan, Ann Arbor, USA \\ ${ }^{3}$ Princess Alexandra Eye Pavilion, Edinburgh, Scotland, UK \\ ${ }^{4}$ The University of Edinburgh, Scotland, UK
}

Received: 24 May, 2017; Accepted: 22 June, 2017; Published: 03 July, 2017

*Corresponding author: V. Swetha E. Jeganathan, Kellogg Eye Center, 1000 Wall Street, Ann Arbor, MI 48105, USA, Phone: +1 (734) 763- 8122; Fax +1 (734) 936-2340; E-mail: vswetha@ausdoctors.net

\begin{abstract}
Context/Background: To identify the proportion of patients with glaucoma presenting to hospital eye services with advanced disease and determine risk factors for late presentation.

Methods: A retrospective study was conducted including100 consecutive patients newly referred from community optometrists to the glaucoma clinic at the Princess Alexandra Eye Pavilion, Edinburgh, Scotland. All subjects underwent slit lamp examination, intraocular pressure (IOP), central corneal thickness (CCT), fundus examination and standard automated perimetry (SAP). 84 patients with glaucoma were categorised according to SAP mean deviation (MD) in the worse eye as 'late presenters' if MD was $\leq-6 \mathrm{~dB}$; and 'very late presenters' if MD was $\leq-10 \mathrm{~dB}$.Logistic regression analysis identified factors associated with late presentation.
\end{abstract}

Results: Mean age was $73.2 \pm 10.2$ years. 36 patients $(42.9 \%)$ were female and $28(33.3 \%)$ had a family history of glaucoma. 41 of 84 $(48.8 \%)$ had an MD in the worse eye $\leq-6 \mathrm{~dB}$ and 23 of $84(27.4 \%)$ had an MD in the worse eye $\leq-10 \mathrm{~dB}$. Older age $(p=0.004)$ was a significant risk factor for late presentation. Gender $(p=0.660)$, IOP $(p=0.712)$, CCT $(p=0.968)$, and family history $(p=0.108)$ were not associated with late presentation.

Conclusions: Half of patients presenting to hospital eye services for the first time with newly diagnosed glaucoma had a MD $\leq-6 \mathrm{~dB}$ in at least one eye. Older patients were more likely to present with advanced disease. Increasing awareness of glaucoma in the elderly population may allow earlier diagnosis and reduce vision related morbidity.

Keywords: Glaucoma; Risk factors; Visual fields; Late presentation; Age

\section{Introduction}

Glaucoma, a chronicoptic neuropathy, is the second most common cause of blindness worldwide [1-3]. As visual loss from glaucoma is irreversible, early diagnosis is important for timely initiation of treatment and preservation of vision [4]. Patients with glaucoma may remain asymptomatic until advanced stages of the disease; therefore, detection of glaucoma often relies on detection of abnormality on routine eye examinations $[5,6]$.

In the UK, free eye examinations are available in primary care with community optometrists. In Scotland, all residents aged over 60 years are entitled to an annual eye examination, in addition to all those over the age of 40 years with a first-degree relative with glaucoma [7]. In those at risk of glaucoma, the NHS eye examination includes tonometry, optic disc examination and assessment of the visual field. If glaucoma is suspected, the patient will be referred to the hospital eye service for further examination with an ophthalmologist, confirmation of the diagnosis and any necessary treatment [8]. Despite the widespread availability of free eye examinations patients continue to present with advanced glaucoma, which is a major concern as advanced glaucoma at presentation is a risk factor for subsequent blindness [5].

Much research has focused on the detection of early glaucoma, however more needs to be done to examine and address the reasons for presenting with advanced disease (late presentation). Previous studies have identified risk factors associated with glaucoma to include older age, thin central corneal thickness (CCT), diabetes and family history of glaucoma in first degree relative [9-15]. Previously identified risk factors for late presentation include black ethnicity, socioeconomic status and older age [10,16-18]. The purpose of this paper was to determine the proportion of patients presenting to hospital services with advanced glaucoma, and examine risk factors for late presentation.

\section{Methods}

\section{Study Population and Data Collection}

We performed a retrospective chart review of 100 consecutive patients with glaucoma or suspected glaucoma referred from community optometrists to the glaucoma clinic at Princess Alexandra Eye Pavilion, Edinburgh, Scotland. The quality improvement panel at Princess Alexandra Eye Pavilion Edinburgh prospectively approved the study methods, and the 
study adhered to the tenets of the Declaration of Helsinki for research involving human subjects. Patients were identified using the electronic referral log to examine consecutive series of referrals to the glaucoma clinic was obtained.

At the first hospital visit all subjects underwent a comprehensive ophthalmological examination including Goldmann applanation tonometry, pachymetry, gonioscopy, dilated fundus examination and optic disc photographs, and visual field testing using the 24-2 SITA-Fast program of the Humphrey Field Analyzer (Carl Zeiss Meditec, Inc., Dublin, California). Those patients with visual fields with more than $33 \%$ fixation losses or more than $15 \%$ false-positive errors, or other are facts at their first visit, had the field test from their subsequent visit analysed. In all cases this was within 6 months of the initial test. If the visual field test from the second visit was also unreliable the patient was excluded from subsequent analysis.

The study included patients with primary open angle glaucoma, pseudo exfoliative glaucoma, pigment dispersion glaucoma, primary angle closure glaucoma and those with suspected glaucoma. The diagnosis of glaucoma was based on the presence of glaucomatous changes to the optic nerve head or retinal nerve fibre layer on fundoscopy or disc photographs by a consultant ophthalmologist with corresponding glaucomatous visual field changes on SAP. The diagnosis of suspect glaucoma was based on the presence of glaucomatous changes to the optic nerve head or retinal nerve fibre layer without changes on SAP. For the analysis, patients were categorised according to SAP mean deviation (MD) in the worse eye. 'Late presenters' were patients with an MD of $\leq-6 \mathrm{~dB}$ in the worse eye, whereas 'very late presenters' had a SAP MD of $\leq-10 \mathrm{~dB}$ in the worse eye.

\section{Statistical Analysis}

Descriptive statistics were used to compare demographic and clinical characteristics of late and non-late presenters. Descriptive statistics yielded mean, standard deviation (SD) and t-tests for normally distributed variables. The Fisher exact test was used to compare categorical variables, and the Wilcoxon rank-sum test was used to compare continuous variables. Logistic regression analysis was used to identify factors associated with late presentation with multivariable analyses used to compare the possible relationship of CCT, IOP and age on severity at presentation. A P-value less than 0.05 was considered statistically significant and statistical analyses were conducted using commercially available statistical software (Stata version 13, StataCorp LP, TX, USA).

\section{Results}

Of the 100 patients selected for this study, 16 patients (19\%) were excluded due to normal examination or a diagnosis of ocular hypertension alone. The remaining 84 patients $(81 \%)$ who were diagnosed with glaucoma or suspect glaucoma were selected for further analysis. All subjects were able to produce at least one reliable visual field.
Table 1: Patient demographics and optic parameters. Age and SAP were associated with late and very late presentation. Values in the second and third columns denote the mean \pm SD.

\begin{tabular}{|l|c|c|c|}
\hline & $\begin{array}{c}\text { Non-late } \\
\text { presenter } \\
(\mathbf{N = 4 3 )}\end{array}$ & $\begin{array}{c}\text { Late } \\
\text { presenter } \\
(\mathbf{n = 4 1 )}\end{array}$ & P-value \\
\hline Age & $70.0 \pm 10.5$ & $76.4 \pm 8.9$ & 0.004 \\
\hline Gender, n=female & $17(39.5 \%)$ & $19(46.3 \%)$ & 0.660 \\
\hline Family history, n=yes & $18(41.9 \%)$ & $10(24.4 \%)$ & 0.108 \\
\hline CCT in worse eye & $546 \pm 37$ & $546 \pm 40$ & 0.968 \\
\hline IOP in worse eye & $20.0 \pm 5.2$ & $20.4 \pm 5.6$ & 0.712 \\
\hline MD in worse eye & $-2.7 \pm 1.7$ & $-13.6 \pm 7.1$ & $<0.001$ \\
\hline
\end{tabular}

Table 1 summarizes the demographic and clinical characteristics of these subjects.

Patients had a mean \pm standard deviation age of $73.2 \pm 10.2$ years.36 of 84 patients (42.9\%) were female and 28 (33.3\%) had a family history of glaucoma in a first or second-degree relative. The average MD in the worse eye at presentation was $-8.03+$ $-7.49 \mathrm{~dB}$ (range -33.46 to $0 \mathrm{~dB}$ ), compared to an average MD in the better eye of $-3.34+-4.39 \mathrm{~dB}$ range -26.07 to $0.76 \mathrm{~dB}$ ). $48.8 \%$ (41 of 84 ) had an MD in the worse eye $\leq-6 \mathrm{~dB}$ (late presenters) and $27.4 \%$ (23 of 84 ) had an MD in the worse eye $\leq 10 \mathrm{~dB}$ (very late presenters).

Older age was a significant risk factor for late presentation $(\mathrm{p}=0.004)$. The mean age for late and non-late presenters was $76.4 \pm 8.9$ years and $70.0 \pm 10.5$ years respectively (Table 1 ). Figure 1 shows that a 10 -year increase in age increased the odds of late presentation by a factor of 1.99 (MD $\leq-6 \mathrm{~dB} ; \mathrm{p}=0.006)$ and increased odds of very late presentation by a factor of 1.66 (MD $\leq-10 \mathrm{~dB} ; \mathrm{p}=0.059$ ).

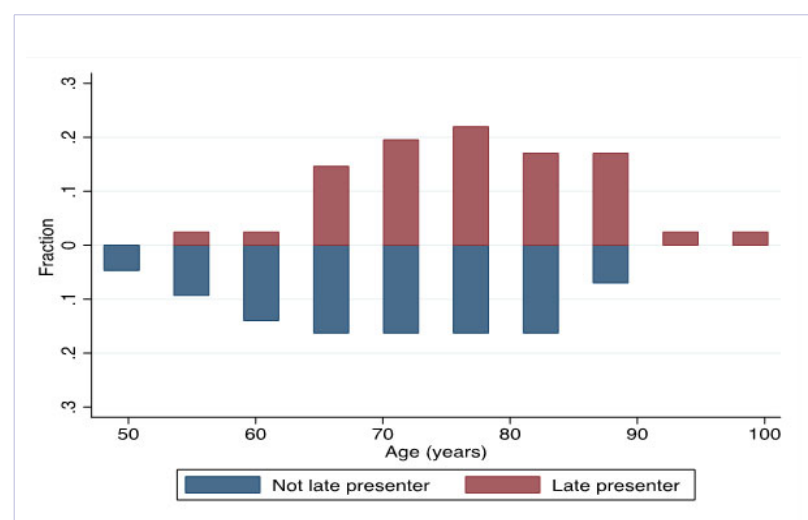

Figure 1:Comparison of Age at presentation between late and non-late presenters

Figure 2 shows that at 60 years of age, probability of late presentation was $20 \%$. At 80 years of age, probability of late presentation had doubled to $40 \%$. 


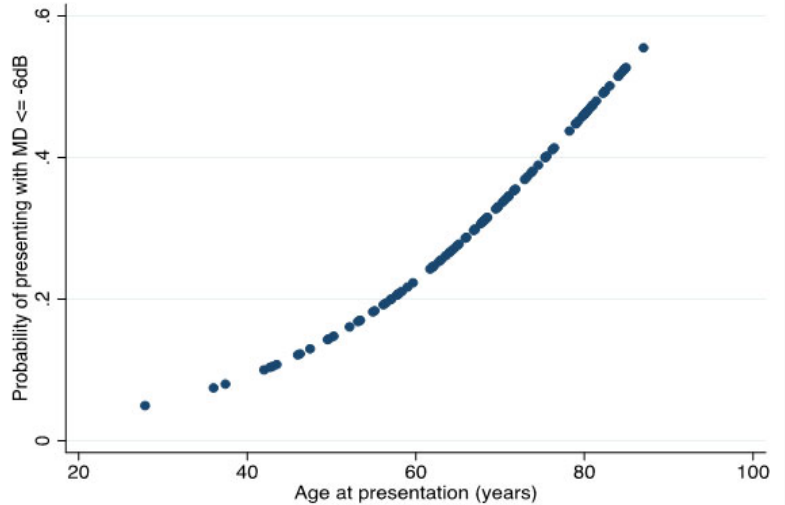

Figure 2: Probability of late presentation using univariable logistic regression.

Of the 41 late presenters, a positive family history was detected in $24.4 \%(n=10)$. There was no association between CCT and late presentation ( $\mathrm{P}=0.968)$, with a similar distribution of CCT amongst late and non-late presenters (Figure 3).

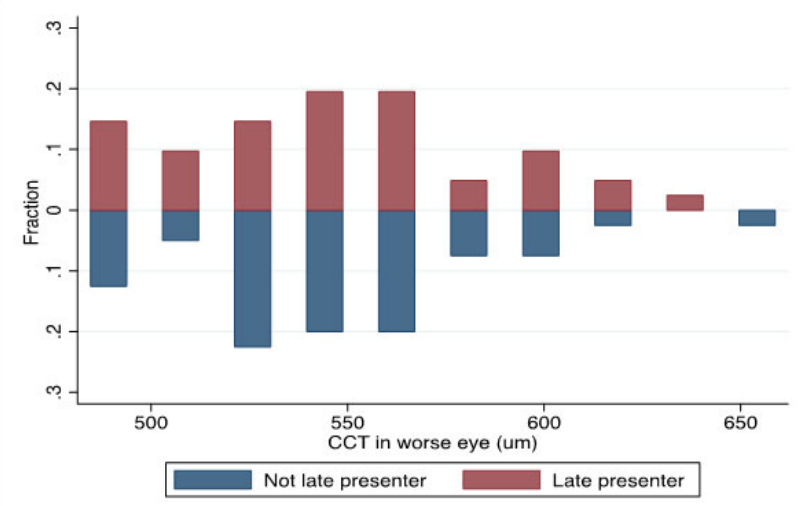

Figure 3: Comparison of CCT at presentation between late and non-late presenters

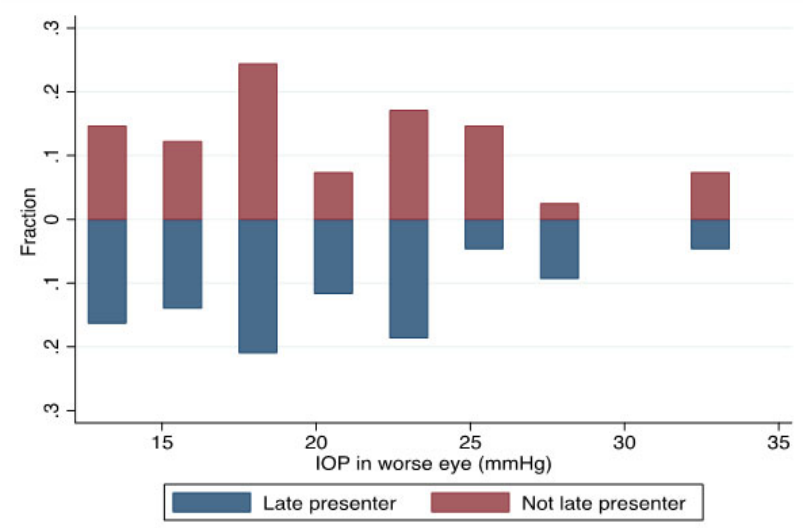

Figure 4: Comparison of IOP at presentation between late and non-late presenters
There was also no association between IOP at presentation and late presentation ( $\mathrm{p}=0.712$ ) (Figure 4). The mean IOP of late and non-late presenters was $20.4 \pm 5.6 \mathrm{mmHg}$ and $20.0 \pm 5.2$ $\mathrm{mmHg}$ respectively.

\section{Discussions}

The results of this study demonstrate that a large proportion of patients diagnosed with glaucoma have significant visual field loss at the time of diagnosis, even in a country where free eye health checks are available. Almost $50 \%$ of patients in this study presented with a MD $\leq-6 \mathrm{~dB}$ in the worse eye and over one quarter of patients presented with a MD $\leq-10 \mathrm{~dB}$ in the worse eye. Previous studies have suggested $10-20 \%$ of patients with glaucoma have advanced visual field loss at presentation and are at high risk for subsequent blindness. This quoted number (10$20 \%$ ) is much lower than our study $(48.8 \%)$. We chose $-6 \mathrm{~dB}$ as a cut of for later presenters in our study, but the definition for advanced glaucoma is somewhat arbitrary.

There are several possible reasons why patients may present late. As glaucoma is usually asymptomatic until there has been substantial loss of visual field, patients who do not have regular eye health checks may not attend their optometrist until they develop symptoms [21]. The other possibility is that patients may be having regular eye examinations but receive a poor level of care, enduring diagnostic and treatment delay [22,23]. The proportion of patients presenting with advanced disease is likely to vary in different countries, depending on the availability of eye care professionals and the awareness of the importance of eye examinations among the population.

We found older age to be a significant risk factor for late presentation, with the odds of late presentation increasing by 2 -fold for each 10 years of increasing age, and the odds of very late presentation increasing by a factor of 1.66. At aged 40, patients had a $10 \%$ chance of presenting late, compared to $20 \%$ chance at age 60 , and just over $40 \%$ chance at age 80 years. As glaucoma is more common with ageing and older patients will have had more time to accumulate damage, it is to be expected that older patients will have a greater likelihood of having advanced disease at presentation. Other reasons for older people presenting with more advanced disease might also include poor awareness of glaucoma or lack of regular eye examination, however it seems probable that older people are likely to be more aware of chronic diseases affecting their peers and more likely to seek regular eye exams $[10,17,21,24]$.

The finding of an association between older age and severity of glaucoma at presentation should not be necessarily be interpreted as meaning resources for glaucoma detection should be directed more favourably at this group. Even though older patients had more advanced disease at presentation, in some cases, due to shorter life expectancy, their life-time risk of visual impairment may be less than younger patients presenting with less severe disease. Also the current system of free eye examinations in Scotland was only introduced 10 years ago and the effects of this initiative may take longer to manifest. Older 
patients presenting with advanced disease now, may have missed out on eye examinations at an earlier age [25].

Prior and colleagues have interviewed late-presenting patients with regards to how and when their glaucoma was detected.[25]One patient stated that they felt their vision was poor due to old age and just needing reading glasses, when in fact the real cause was glaucoma. Therefore, some patients may attribute visual loss due to glaucoma to "inevitable ageing" and may confuse signs with presbyopia. Another patient recounted that they were delays in referral from their general practitioner to the ophthalmologist. It had taken 16 weeks for the patient to be seen by a specialist and it was then that they were diagnosed with severe glaucoma. This reinforces the importance of educating both health professionals and patients with regards, to the presentation of glaucoma. In our study, optometrists referred all of the glaucoma suspects to the glaucoma specialist. Patients referred by a source other than their optometrist (e.g. GPs) are 4-5 times more likely to attend with late stage disease $[9,20]$. Investigating a correlation between late stage disease and referral source would provide more information regarding the obstacles our healthcare system faces in light of the increasing number of severe cases of glaucoma at first presentation.

The role of a high IOP and thinner CCT as risk factors in the development of glaucoma are well established however, we found no association between CCT or IOP and late presentation $[11,12]$. This is somewhat surprising as one might expect eyes with lower IOP to present late due to the historical emphasis placed on raised IOP in glaucoma case finding. Previous literature has suggested high IOP to best wrongly associated with late presentation of glaucoma $[26,28]$. A previous study by Jay and colleagues found that patients with untreated glaucoma presenting with IOPs greater than $21 \mathrm{mmHg}$ were likely to progress to end stage visual loss in 14 years and those with initial pressures of greater than 30 $\mathrm{mmHg}$ likely to progress to end stage visual loss in only 3 years [27]. It might be that patients in our study are screened more thoroughly for IOPs and are referred sooner to specialists. Patients in other countries may have to be referred from optometrists to GPs or directly from the GPs with the intention of forward referral to an ophthalmologist. This may delay attendance and lead to later disease at presentation, which was commented on by other studies. Furthermore, it is very rare for the GPs to have the equipment or expertise to examine patients for glaucoma.

We found no association between family history of glaucoma and late presentation, in agreement with a previous study based in South Africa [28]. In contrast, Prior and colleagues found that those with a positive family history of glaucoma were less likely to present late than those with no family history whilst a third study found patients with a positive family history of glaucoma were more likely to present with advanced disease $[17,25]$. The association between family history and late presentation therefore seems somewhat variable and the risk of late presentation probably depends more on patient awareness of glaucoma risk and on other parameters such age, education and socioeconomic status $[10,17,18]$.
The current study has some limitations. The sample size in this retrospective study is relatively small compared to other studies. For example, Buys et al analysed 290 patients in their study and Fraser et al had a sample size of 200 patients $[9,18]$. Furthermore, we believe that not all risk factors for late presentation were identified in this study. Although, we investigated the association between IOP, CCT, family history, gender, and age, other studies have identified other factors for late presentation such as lower socioeconomic status, African-Caribbean ethnicity, method of referral to hospital other than optometrist, and longer period of time since last visit to optometrist [10,16-18,21]. It would be particularly important to investigate a potential link between socio-economic status and late presentation, as this is a potentially confounding factor in the interpretation of our results and the finding of a relationship between lower socio-economic status and late presentation would support the need to introduce interventions to break down any barriers associated with access to eye care services in areas of higher deprivation [29].

\section{Conclusions}

In conclusion, although most patients with newly diagnosed glaucoma had early or moderate disease at presentation, almost half $(48.8 \%)$ had an MD of $\leq-6 \mathrm{~dB}$ in at least one eye and older patients were more likely to present late. Efforts to increase awareness of glaucoma may reduce vision related morbidity and allow earlier diagnosis.

\section{Acknowledgements}

\section{Declarations}

Conflict of interest: A.J.T. - Funding from Allergan and Thea

Funding: Supported by a grant from Edinburgh and Lothian's Health Foundation and a NHS Scotland Career Research Fellowship (AJT)

Ethical approval: Ethics approval for the study was obtained from the quality improvement panel at Princess Alexandra Eye Pavilion Edinburgh prospectively, and the studyconforms to the provisions of the Declaration in Helsinki. Consent was obtained from all patients before enrolment and their anonymity was preserved.

\section{References}

1. Weinreb RN, Khaw PT. Primary open-angle glaucoma. Lancet. 2004;363(9422):1711-1720.

2. Kingman S. Glaucoma is second leading cause of blindness globally. Bull World Health Organ. 2004;82(11):887-888.

3. Quigley HA, Broman AT. The number of people with glaucoma worldwide in 2010 and 2020. Br J Ophthalmol. 2006;90(3):262-267.

4. Bettin P, Di Matteo F. Glaucoma: present challenges and future trends. Ophthalmic Res. 2013;50(4):197-208. doi: 10.1159/000348736

5. de Moraes CG, Liebmann JM, Medeiros FA, Weinreb RN. Management of advanced glaucoma: Characterization and monitoring. Surv Ophthalmol. 2016;61(5):597-615. doi: 10.1016/j. survophthal.2016.03.006 
6. Dervisevic E, Pavljasevic S, Dervisevic A, Kasumovic SS. Challenges In Early Glaucoma Detection. Med Arch. 2016;70(3):203-207. doi: 10.5455/medarh.2016.70.203-207

7. http://www.gov.scot/Publications/2010/08/18091434/1 .

8. Fearns N, Graham K, Johnston G, Service D. Improving the user experience of patient versions of clinical guidelines: user testing of a Scottish Intercollegiate Guideline Network (SIGN) patient version. BMC Health Serv Res. 2016;16:37. doi: 10.1186/s12913-016-12878

9. Fraser S, Bunce C, Wormald R. Retrospective analysis of risk factors for late presentation of chronic glaucoma. $\mathrm{Br} \mathrm{J}$ Ophthalmol. 1999;83(1):24-28.

10. Fraser S, Bunce C, Wormald R. Risk factors for late presentation in chronic glaucoma. Invest Ophthalmol Vis Sci. 1999;40(10):22512257.

11. Salvetat ML, Zeppieri M, Tosoni C, Brusini P; Medscape. Baseline factors predicting the risk of conversion from ocular hypertension to primary open-angle glaucoma during a 10-year follow-up. Eye (Lond). 2016;30(6):784-795. doi: 10.1038/eye.2016.86

12. Lee BL, Wilson MR, Ocular Hypertension Treatment Study (OHTS). Ocular Hypertension Treatment Study (OHTS) commentary. Curr Opin Ophthalmol. 2003;14(2):74-77.

13. Zhou M, Wang W, Huang W, Zhang X. Diabetes mellitus as a risk factor for open-angle glaucoma: a systematic review and meta-analysis. PLoS One. 2014;9(8):e102972. doi: 10.1371/journal.pone.0102972

14. Gupta D, Chen PP. Glaucoma. Am Fam Physician 2016;93(8):668674.

15. Gong G, Kosoko-Lasaki S, Haynatzki G, Lynch HT, Lynch JA, Wilson MR. Inherited, familial and sporadic primary open-angle glaucoma. J Natl Med Assoc. 2007;99(5):559-563.

16. Ntim-Amponsah CT, Amoaku WM, Ewusi RK, Idirisuriya-Khair R, Nyatepe-Coo E, Ofosu-Amaah S. Evaluation of risk factors for advanced glaucoma in Ghanaian patients. Eye (Lond). 2005;19(5):528-534.

17. Fraser S, Bunce C, Wormald R, Brunner E. Deprivation and late presentation of glaucoma: case-control study. BMJ. 2001;322(7287):639-643.
18. Buys YM, Jin YP, Canadian Glaucoma Risk Factor Study Group. Socioeconomic status as a risk factor for late presentation of glaucoma in Canada. Can J Ophthalmol. 2013;48(2):83-87. doi: 10.1016/j.jcjo.2012.10.003

19. Coffey M, Reidy A, Wormald R, Xian WX, Wright L, Courtney P. Prevalence of glaucoma in the west of Ireland. Br J Ophthalmol. 1993;77(1):17-21.

20. Sheldrick JH, Ng C, Austin DJ, Rosenthal AR. An analysis of referral routes and diagnostic accuracy in cases of suspected glaucoma. Ophthalmic Epidemiol. 1994;1(1):31-39.

21. Sukumar S, Spencer F, Fenerty C, Harper R, Henson D. The influence of socioeconomic and clinical factors upon the presenting visual field status of patients with glaucoma. Eye (Lond). 2009;23(5):10381044. doi: 10.1038/eye.2008.245

22. Hitchings RA. Glaucoma screening in the elderly. Curr Med Res Opin. 1982;7(Suppl 1):23-7.

23. Fleming C, Whitlock E, Beil T, Smit B. Primary Care Screening for Ocular Hypertension and Primary Open-Angle Glaucoma. Rockville (MD), 2005.

24. Ng WS, Agarwal PK, Sidiki S, McKay L, Townend J, Azuara-Blanco A. The effect of socio-economic deprivation on severity of glaucoma at presentation. Br J Ophthalmol. 2010;94(1):85-87. doi: 10.1136/ bjo.2008.153312

25. Guedes G, Tsai JC, Loewen NA. Glaucoma and aging. Curr Aging Sci. 2011;4(2):110-117.

26. Prior M, Francis JJ, Azuara-Blanco A, Anand N, Burr JM; Glaucoma screening Platform Study group. Why do people present late with advanced glaucoma? A qualitative interview study. Br J Ophthalmol. 2013;97(12):1574-1578. doi: 10.1136/bjophthalmol-2013-303813

27. Jay JL, Murdoch JR. The rate of visual field loss in untreated primary open angle glaucoma. Br J Ophthalmol. 1993;77(3):176-178.

28. Kraukamp P. Factors associated with late presentation of glaucoma: A study in a black South African population (Doctoral dissertation, Faculty of Health Sciences, University of the Witwatersrand, Johannesburg). 2013.

29. Trudinger D NV. The barriers and enablers that affect access to primary and secondary eye care services-Hackney site report. London: RNIB, 2012. Report No.: RNIB/CEP/IR/Hackney/01. 